\title{
Mechanisms of WNK1 and WNK4 interaction in the regulation of thiazide-sensitive $\mathrm{NaCl}$ cotransport
}

\author{
Chao-Ling Yang, ${ }^{1,2}$ Xiaoman Zhu, ${ }^{1,2}$ Zhaohong Wang, ${ }^{1}$ \\ Arohan R. Subramanya, ${ }^{1}$ and David H. Ellison ${ }^{1,2,3}$
}

\begin{abstract}
'Division of Nephrology and Hypertension, Oregon Health \& Science University, Portland, Oregon, USA. 2Portland Veterans Affairs Medical Center, Portland, Oregon, USA. ${ }^{3}$ Department of Physiology and Pharmacology, Oregon Health \& Science University, Portland, Oregon, USA.
\end{abstract}

\begin{abstract}
With-no-lysine (WNK) kinases are highly expressed along the mammalian distal nephron. Mutations in either WNK1 or WNK4 cause familial hyperkalemic hypertension (FHHt), suggesting that the protein products converge on a final common pathway. We showed previously that WNK4 downregulates thiazide-sensitive $\mathrm{NaCl}$ cotransporter (NCC) activity, an effect suppressed by WNK1. Here we investigated the mechanisms by which WNK1 and WNK4 interact to regulate ion transport. We report that WNK1 suppresses the WNK4 effect on NCC activity and associates with WNK4 in a protein complex involving the kinase domains. Although a kinase-dead WNK1 also associates with WNK4, it fails to suppress WNK4-mediated NCC inhibition; the WNK1 kinase domain alone, however, is not sufficient to block the WNK4 effect. The carboxyterminal 222 amino acids of WNK4 are sufficient to inhibit NCC, but this fragment is not blocked by WNK1. Instead, WNK1 inhibition requires an intact WNK4 kinase domain, the region that binds to WNK1. In summary, these data show that: (a) the WNK4 carboxyl terminus mediates NCC suppression, (b) the WNK1 kinase domain interacts with the WNK4 kinase domain, and (c) WNK1 inhibition of WNK4 is dependent on WNK1 catalytic activity and an intact WNK1 protein. These findings provide insight into the complex interrelationships between WNK1 and WNK4 and provide a molecular basis for FHHt.
\end{abstract}

\section{Introduction}

Familial hyperkalemic hypertension (FHHt; also known as pseudohypoaldosteronism type II; Online Mendelian Inheritance in Man reference number\#145260) is an autosomal dominant disease characterized by hypertension, hyperkalemia, and sensitivity to thiazide diuretics. Wilson and colleagues (1) reported that mutations in 2 genes encoding homologous proteins, WNK1 (PRKWNK1) and WNK4 (PRKWNK4), can cause FHHt. The with-no-lysine (WNK) kinases are novel serine/threonine kinases that were named because they lack lysine at a location previously thought to be essential for kinase activity (2). Both WNK1 and WNK4 are highly expressed in the kidney. The FHHt-causing WNK1 mutations are deletions within the first intron. These mutations increase WNK1 expression in leukocytes and were postulated to be gain-of-function mutations (1). Recently, heterozygous WNK1-deficient mice were shown to exhibit lower blood pressure than wild-type controls (3), supporting the hypothesis that WNK1 exerts a positive effect on blood pressure. The WNK4 mutations that cause FHHt are discrete missense mutations in 2 areas of the coding region (1). These mutations cause phenotypic features similar to those that result from WNK1 intron mutations (1), but the mechanisms involved may be different.

We showed previously that WNK4 inhibits the thiazide-sensitive $\mathrm{NaCl}$ cotransporter (NCC, gene symbol SLC12A3) when expressed in Xenopus oocytes (4); similar results were obtained by others (5).

Nonstandard abbreviations used: FHHt, familial hyperkalemic hypertension; NCC, thiazide-sensitive $\mathrm{NaCl}$ cotransporter; ROMK, inwardly rectifying $\mathrm{K}$ channel; WNK, with no lysine.

Conflict of interest: The authors have declared that no conflict of interest exists.

Citation for this article: J. Clin. Invest. 115:1379-1387 (2005).

doi:10.1172/JCI200522452.
In contrast, WNK1 did not affect NCC activity directly, but instead strongly suppressed the WNK4 effect on NCC (4); in other words, WNK1 coexpression with WNK4 returned NCC activity close to baseline levels. The mechanisms by which WNK kinases interact were not elucidated by these experiments. Both kinase-dependent and kinaseindependent effects may be involved, as some $(2,6-8)$, but not all $(7,9-11)$, WNK kinases possess kinase activity. Furthermore, all WNK kinases contain coiled-coil domains that may participate in proteinprotein interactions. The current experiments investigated mechanisms by which WNK1 and WNK4 interact to regulate NCC activity. The results demonstrate that the carboxyl terminus of WNK4 is sufficient to inhibit NCC but is not blocked by WNK1. WNK1-mediated WNK4 suppression is dependent on both binding and catalytic activity. These results provide insight into mechanisms by which WNK kinases converge on a common ion transport pathway in the distal nephron and indicate that there are novel protein-protein interactions between different members of this kinase family. They also suggest the potential development of novel antihypertensive agents.

\section{Results}

NCC belongs to the cation chloride cotransporter gene family (SLC12). The mouse NCC comprises 1,001 amino acid residues, including 12 putative membrane-spanning domains, a 132-residue cytoplasmic amino terminus, and a 400-amino acid cytoplasmic carboxyl terminus. WNK kinases comprise novel serine/threonine kinases that share approximately $70 \%$ amino acid identity within their kinase domains. They all appear to possess a short amino-terminal domain and a longer carboxyl terminus, which includes 2 coiled-coil domains. We previously reported that WNK4 downregulates NCC activity and that WNK1 suppresses this effect (4). These effects reflect changes in NCC trafficking to the plasma 

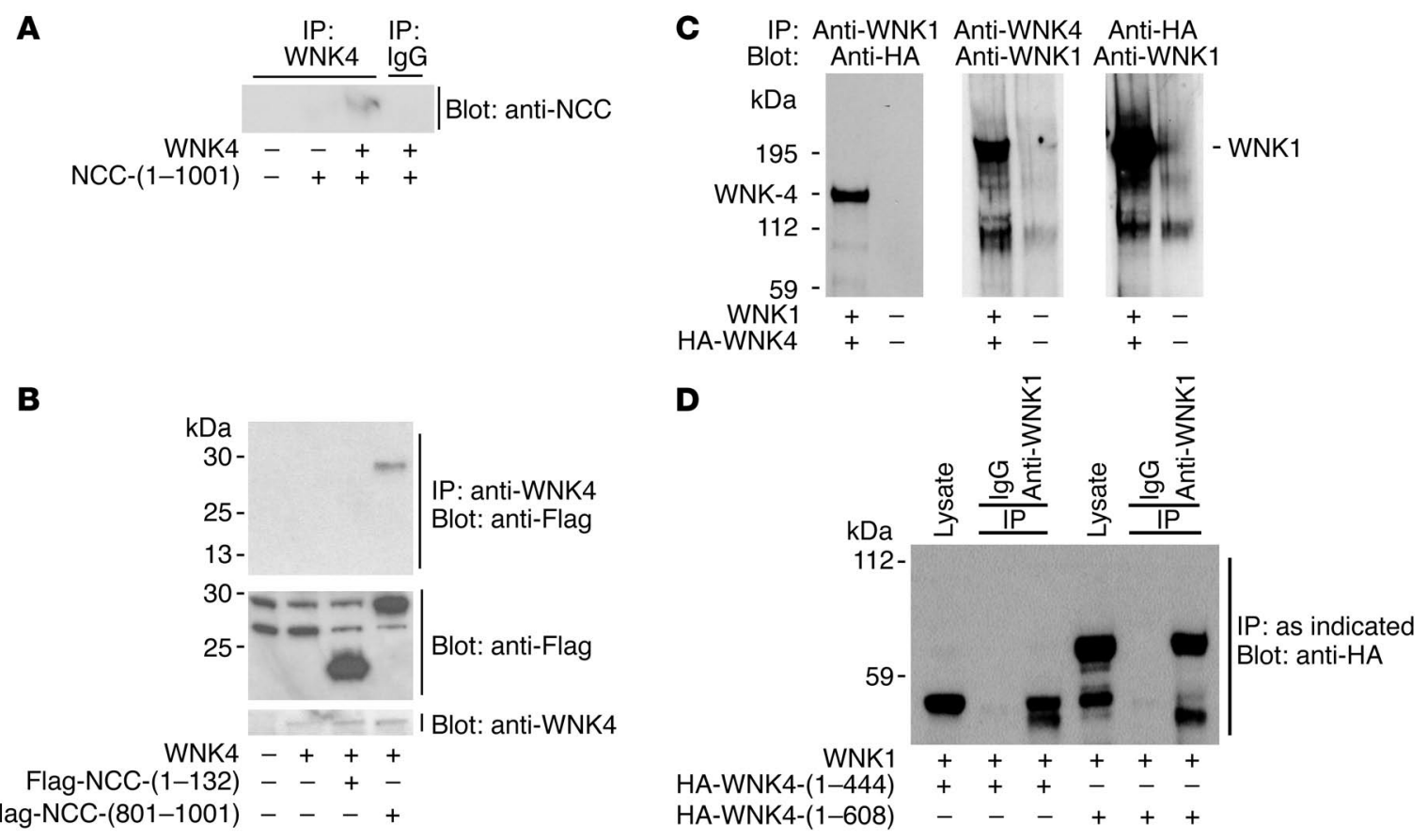

\section{Figure 1}

WNK4 associates with NCC and with WNK1. (A) HEK293 cells were transfected with full-length WNK4 with or without full-length NCC. Lysates were immunoprecipitated with anti-WNK4 or a control IgG. An immunoprecipitated product was present only when both NCC and WNK4 were included. Shown is 1 of 3 similar experiments. (B) HEK293 cells were transfected with Flag-tagged NCC-(1-132) or Flag-tagged NCC-(801-1001) and WNK4. Lysates were immunoprecipitated using anti-WNK4. Immunoblots of cell lysates, detected with anti-Flag and anti-WNK4 (bottom), indicate expression of the expected proteins. Nonspecific bands appear in all lanes (middle). WNK4 immunoprecipitated NCC-(801-1001) but not NCC-(1-132) (top). Shown is 1 of 3 similar experiments. (C) Oocytes injected with cRNA encoding full-length WNK1, HA-WNK4, or water were subjected to immunoprecipitation with anti-WNK1, anti-WNK4, or anti-HA, and then blotted with anti-HA or antiWNK1 as indicated. WNK4 was immunoprecipitated by WNK1. WNK1 was immunoprecipitated by both anti-WNK4 and anti-HA. Shown is 1 of 3 similar experiments. (D) Immunoprecipitation of WNK4 fragments by full-length WNK1. Oocytes were injected with WNK1 and the indicated HA-tagged WNK4. The blot shows cell lysates or material immunoprecipitated with either IgG (control) or anti-WNK1. The results show that both WNK4 fragments are precipitated by WNK1. Shown is 1 of 3 similar experiments.

membrane. Here we examined whether the functional effects reflect protein-protein interactions and, if so, what protein domains interact to determine physiological effects of WNK kinases.

WNK4 associates with NCC and WNK1. We demonstrate that fulllength WNK4 interacted with full-length NCC when expressed in HEK293T cells and confirm results of others (5) showing further that WNK4 could associate with the carboxyterminal 200 amino acid residues of NCC (Figure 1, A and B). Although both aminoterminal (NCC-[1-132]) and carboxyterminal (NCC-[801-1001]) constructs were expressed at the protein level in HEK293T cells, only the carboxyterminal NCC fragment, and not the amino-terminal fragment, was precipitated with anti-WNK4 (Figure 1B).

We showed previously (4) that WNK1 could largely suppress WNK4-mediated NCC inhibition when expressed in Xenopus oocytes. In the current experiments, we used the same experimental system to determine whether WNK1 and WNK4 could associate in a protein complex. WNK1 precipitated WNK4, and vice versa, utilizing both anti-WNK4 and anti-HA antibodies (Figure 1C).

To identify the WNK4 domains responsible for interacting with WNK1, immunoprecipitation experiments were performed using full-length WNK1 and truncated WNK4 constructs. Both WNK4-(1-444) and WNK4-(1-608) interacted with WNK1 (Figure 1D), indicating that the amino-terminal WNK4 kinase domain interacts with WNK1.
A kinase-dead WNK1 associates with, but does not inhibit, WNK4. WNK1 is a catalytically active kinase $(2,7,8)$. Cobb and colleagues demonstrated that the WNK1-(D368A) mutant is catalytically inactive (2). We examined whether WNK1 catalytic activity is necessary for its effects on WNK4. Figure 2A confirms our previous observations that WNK4 inhibits NCC activity and that coexpression with WNK1 suppresses the WNK4 effect (i.e., WNK1 inhibits WNK4). In contrast, coexpression of WNK1-(D368A) with WNK4 did not restore NCC activity to baseline levels. Therefore, kinase-dead WNK1 does not suppress WNK4 inhibition of NCC. We then tested whether WNK1-(D368A) associates with WNK4 in a protein complex, as wild-type WNK1 does. Figure 2B shows that both wild-type WNK1 and WNK1-(D368A) associated with WNK4 in a protein complex when expressed in Xenopus oocytes. This suggests that kinase-dead WNK1 does not lose its inhibiting activity simply because it does not bind to WNK4. Additional experiments will be needed to determine whether WNK1 phosphorylates WNK4 directly.

The WNK4 carboxyl terminus inbibits NCC but does not interact with WNK1. To determine whether the WNK4 kinase domain is required to regulate NCC activity, WNK4 constructs that include the kinase domain (WNK4-[1-444] and WNK4-[168-1222]) and WNK4 constructs that do not include the kinase domain (WNK4[445-1222]) were compared in the Na uptake assay. WNK4 constructs that include the carboxyl terminus inhibited NCC activity 


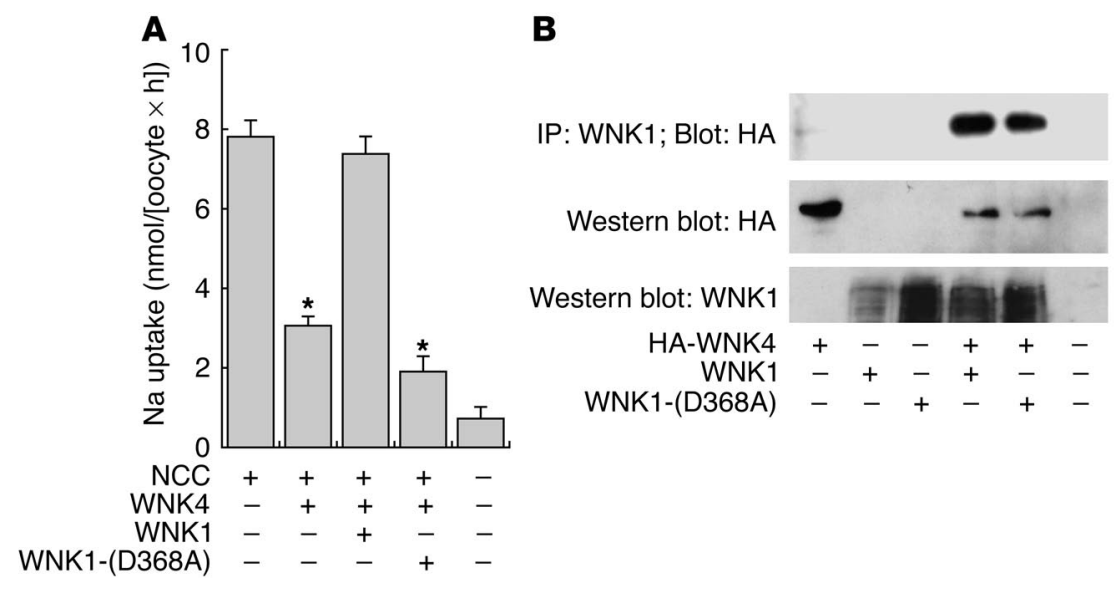

Figure 2

WNK1 catalytic activity is required to inhibit, but not to bind, WNK4. (A) ${ }^{22} \mathrm{Na}$ uptake by oocytes injected with NCC, WNK4, wild-type WNK1, or kinase-deficient WNK1 (WNK1-[D368A]). WNK4 inhibited NCC-mediated Na uptake; WNK1 suppressed the inhibition; WNK1-(D368A) did not suppress WNK4-mediated NCC inhibition. ${ }^{*} P<0.05$ versus NCC alone. (B) Oocytes injected with wild-type or kinase-deficient WNK1 constructs and HA-WNK4 were lysed and precipitated with anti-WNK1. Blots of lysates show protein expression in expected lanes. Both wild-type WNK1 and kinase-deficient WNK1 (WNK1-[D368A]) immunoprecipitated WNK4.

terminal domain, WNK4-(808-1222) and WNK4-(1000-1222), fully inhibited NCC activity. As was the case for the longer carboxyterminal WNK4 construct, however, NCC inhibition by shorter carboxyterminal WNK4 constructs was insensitive to suppression by WNK1 (Figure 4A). These results identify a WNK4 domain that comprises the carboxyterminal 222 amino acids and includes the second coiled-coil domain as sufficient for NCC inhibition. Although this short segment could inhibit NCC, it did not interact with WNK1 functionally (i.e., WNK1 did not suppress its inhibitory effect on WNK4) (Figure 4A). To determine whether WNK4 domains that inhibit NCC associate with the transport protein in a complex, additional immunoprecipitation experiments were performed. The carboxyterminal WNK4 fragments that inhibited NCC strongly associated with the transporter (Figure 4B). In contrast, an ineffective amino-terminal WNK4 fragment associated much less avidly. Figure 4C indicates that the inability of WNK1 to block NCC inhibi-

(Figure 3A). In contrast, the WNK4 construct that comprises only the kinase domain plus the short amino terminus, WNK4-(1-444), did not affect Na uptake. Although this indicates that the WNK4 carboxyl terminus, and not the kinase domain, is essential for NCC inhibition, the results with WNK1 coexpression were strikingly different. Whereas WNK1 suppressed the effect of WNK4-(168-1222) on NCC activity, it had no effect on WNK4-(445-1222)-mediated NCC suppression (Figure 3A). Therefore, the carboxyl terminus of WNK4 is essential for NCC inhibition, but the amino terminus of WNK4, which contains the kinase domain, is essential for WNK1 to suppress the WNK4 effect. Figure 3B shows that WNK1-(1-555) strongly immunoprecipitated WNK4-(1-444) but minimally immunoprecipitated WNK4-(445-1222). Therefore, the amino terminus of WNK1 associates in a protein complex with the amino terminus of WNK4 when expressed in oocytes.

To narrow the regions of WNK kinase interaction further, additional immunoprecipitation experiments were performed. The results, shown in Figure 3C, indicate that the kinase domain of WNK1 (myc-WNK1-[218-491]) specifically immunoprecipitated the WNK4 kinase domain (for example, HA-WNK4-[1-444]) but not protein fragments that contain only the amino-terminal pre-kinase domain or the carboxyl terminus of WNK4. These results indicate that the kinase domains of WNK1 and WNK4 associate with each other.

The results shown in Figure 3A indicate that a WNK4 region distal to the kinase domain is sufficient to inhibit NCC activity. To determine whether other WNK4 domains can inhibit NCC activity and to narrow the NCC inhibitory domain further, additional WNK4 constructs were generated and expressed. WNK4-(1-608) contains the amino terminus, the kinase domain, the autoinhibitory domain, and the first coiled-coil domain. It also contains sites of amino acid mutations in patients with FHHt (1). This construct did not affect NCC-mediated $\mathrm{Na}$ uptake when coexpressed in oocytes with NCC (see Figure 4A). In contrast, WNK4 constructs containing only the far carboxy- tion mediated by WNK4-(1000-1222) can not be explained on the basis of deficient WNK1 protein expression; both WNK4 constructs and WNK1 were expressed by the oocytes.

Localization of a negative regulatory signal region of WNK4. To narrow sites of NCC inhibition further, the ability of a carboxyterminal WNK4 construct to inhibit NCC activity was compared with that of a construct missing the carboxyterminal 47 amino acids. Figure 5A shows that the truncated construct (WNK4-[445-1175]) did not inhibit NCC activity, in contrast to the construct containing the carboxyterminal 47 amino acids (WNK4-[445-1222]). This identifies these amino acids as essential for inhibition. Interestingly, this region does not include a nearby amino acid shown to be mutated in some patients with FHHt (1). To determine whether the diseasecausing WNK4 mutation at this site abrogates NCC-inhibiting activity, this mutant was tested. WNK4-(445-1222)-R1164C was as effective at inhibiting NCC activity as WNK4-(445-1222). Figure 5A also compares this essential WNK4 regulatory domain from three species and with the carboxyterminal 50 amino acids of WNK1.

In view of the identification of the carboxyterminal domain as essential for WNK4's inhibiting activity, we then tested whether these WNK4 constructs interacted with NCC in a protein complex. Immunoprecipitation experiments (Figure $5 \mathrm{~B}$ ) showed that both WNK4-(445-1222) and WNK4-(445-1175) interacted with NCC in a protein complex. Control experiments in which a nonspecific IgG was employed to immunoprecipitate showed that the results were specific. In combination, the results of the functional studies and the immunoprecipitation studies suggest the presence of discrete NCC-binding and NCC-regulatory WNK4 domains.

Catalytically active full-length WNK1 inbibits WNK4-mediated NCC inbibition. In view of the fact that WNK1 kinase activity is essential for WNK1 to inhibit WNK4, we tested whether WNK1 fragments containing the kinase domain alone could block the WNK4 effect. Surprisingly, Figure 6A shows that only a fulllength WNK1 construct inhibited WNK4 mediated suppression of NCC activity. Neither fragments comprising the kinase 


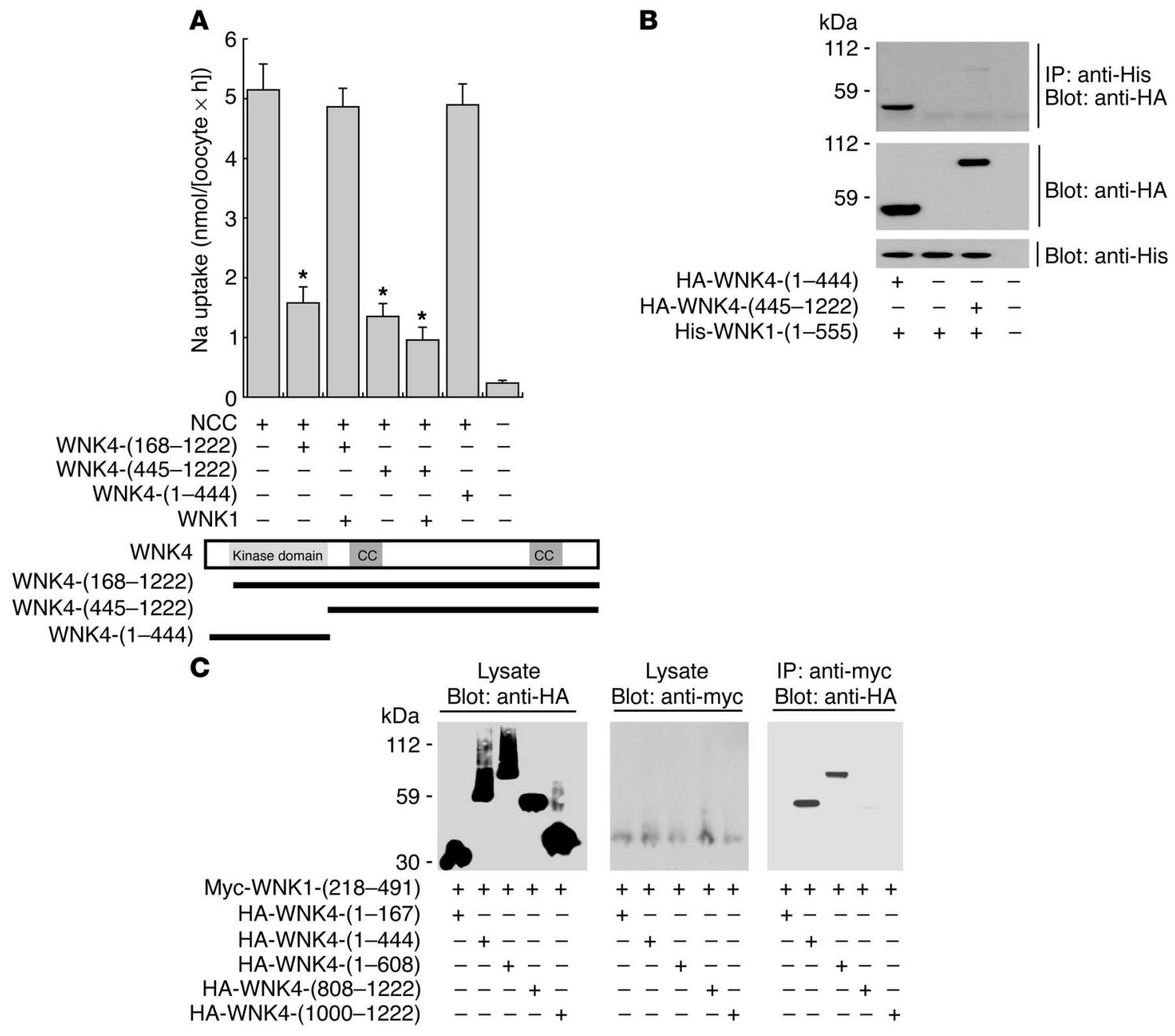

Figure 3

The amino-terminal kinase domain of WNK4 is not required for NCC inhibition but is required for WNK1 interaction. (A) ${ }^{22}$ Na uptake by oocytes injected with cRNA encoding NCC and several WNK4 constructs, with or without WNK1. The WNK4 constructs are diagrammed below. Note that carboxyterminal WNK4 constructs (WNK4-[168-1222] and WNK4-[445-1222]) inhibited Na uptake compared with NCC alone, but a kinasedomain WNK4 construct (WNK4-[1-444]) did not. WNK1 blocked the effect of a WNK4 construct that contains both the kinase domain and the carboxyl terminus (WNK4-[168-1222]) but did not affect the inhibition by carboxyterminal constructs (WNK4-[445-1222]). ${ }^{*}<0.05$ versus NCC alone. cc, coiled coil domains. (B) Immunoprecipitation of the amino-terminal WNK4 domain by WNK1. Oocytes were injected with HA-WNK4 amino- and carboxyterminal constructs and with His-WNK1-(1-555). Only the amino-terminal WNK1 precipitated the amino-terminal WNK4 construct. Bottom blots confirm protein expression of all constructs. A faint band indicates that a much weaker association between the carboxyl terminus of WNK4 and the amino terminus of WNK1 could be detected. (C) Immunoprecipitation of WNK4 fragments by WNK1 kinase domain alone. The left 2 panels show expression of both protein constructs. The right panel shows that myc-WNK1-(218-491) precipitated only WNK4 constructs that contain the kinase domain. Shown is 1 of 3 similar experiments.

domain alone (WNK1-[1-491]) (7), fragments containing the first coiled-coil domain (WNK1-[1-1036]), nor fragments lacking the kinase domain (WNK1-[640-2126]) were able to block WNK4-mediated NCC suppression. Figure 6B confirms that all WNK1 constructs that did not block the WNK4 effect were expressed at the protein level.

A putative kinase-dead WNK4 was reported to lack NCC-inhibiting activity (5). In light of the current results showing NCC inhibition by the carboxyl terminus of WNK4, we tested whether WNK4(D318A)-(168-1222) could inhibit NCC. WNK4-(168-1222) was shown to actively suppress NCC in Figure 3A. The results (Figure 6A) show that WNK4-(D318A)-(168-1222) inhibited NCC as effectively as did wild-type WNK4.

\section{Discussion}

WNK kinases comprise a recently identified group of serine and threonine kinases in which the location of the lysine required for ATP binding is unique (2). Mutations in the genes encoding 2 WNKs, WNK1 and WNK4, cause FHHt in humans, indicating that these kinases play essential roles in regulating renal electrolyte homeostasis (1). WNK kinases are expressed in multicellular organisms, including plants, Caenorhabditis elegans, and Drosophila, but not in the unicellular Saccharomyces cerevisiae (1, 2, 12-14). Mammalian WNK kinases are expressed by epithelial tissues including the distal nephron of mammalian kidney, where they are believed to regulate solute movement $(1,15)$. Although the substrates and physiological functions of WNK kinases remain subjects of ongoing investi- 


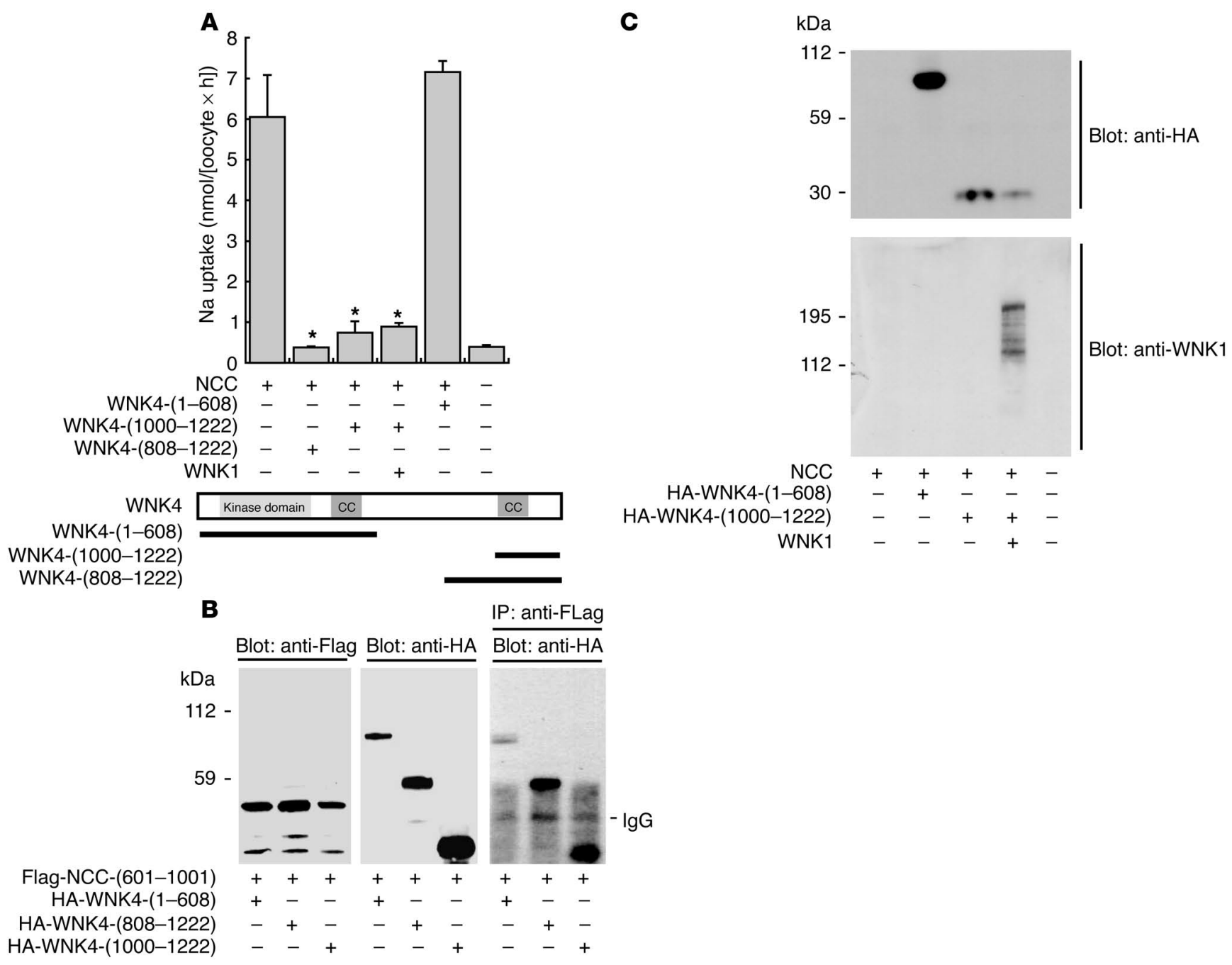

\section{Figure 4}

A short carboxyterminal WNK4 domain binds to and mediates NCC suppression. (A) ${ }^{22}$ Na uptake by oocytes injected with cRNA encoding NCC and several WNK4 constructs, with or without WNK1. The WNK4 constructs are shown diagrammatically. WNK4 constructs that include a short carboxyterminal domain inhibited NCC activity. Note that inhibition by the carboxyl terminus of WNK4 (WNK4-[1000-1222]) was as potent as inhibition by full-length WNK4 (compare WNK4 in Figure 2A with WNK4-[808-1222]). This inhibition was also not suppressed by WNK1. The WNK4 construct containing the kinase domain and the first coiled-coil domain (WNK4-[1-608]) did not inhibit NCC. ${ }^{*} P<0.05$ versus NCC alone. (B) Immunoprecipitation of the NCC carboxyl terminus by WNK4. Oocytes were injected with RNA encoding HA-tagged WNK4 constructs and a Flag-tagged NCC carboxyterminal domain. Although modest interaction between the amino-terminal WNK4 domain and NCC was detected, much stronger interaction between NCC carboxyterminal domain and WNK4 carboxyterminal domain was observed. Shown is 1 of 3 similar experiments. (C) Western blots showing that WNK1 and each of the WNK4 constructs were expressed, both alone and together. The WNK1 antibody used for this blot was Y-1606.

gation, progress in understanding the role they play in regulating epithelial solute transport has been made. When expressed using heterologous expression systems, WNK4 inhibits NCC function by reducing NCC abundance at the plasma membrane (4, 5). WNK4 also reduces the membrane abundance of the inwardly rectifying $\mathrm{K}$ channel (ROMK) (16) and other transport proteins (17), which suggests a general role for WNK4 in regulating epithelial solute transport. The effect of WNK4 to reduce NCC activity has been reported to depend on its kinase activity (5), whereas the effect of WNK4 to reduce the membrane abundance of ROMK (16). Recently, WNK4 has been shown to regulate paracellular ion permeability, perhaps by interacting with claudins $(18,19)$.
WNK1 and WNK4 are both expressed by distal convoluted tubule and connecting tubule cells in the kidney $(1,9)$. The clinical phenotypes of FHHt resulting from mutations of WNK1 and WNK4 are similar $(1,20,21)$. Together, these findings suggest that WNK1 and WNK4 converge on a common pathway in the kidney that regulates blood pressure and electrolyte homeostasis. We showed previously that WNK1, though unable by itself to inhibit NCC activity, suppresses the effects of WNK4 on NCC (4). This indicates that WNK kinases interact and suggests a potential mechanism underlying the final common pathway effects of these distinct kinases. The molecular mechanisms by which WNK kinases regulate ion transport proteins are unknown, although it has been reported 

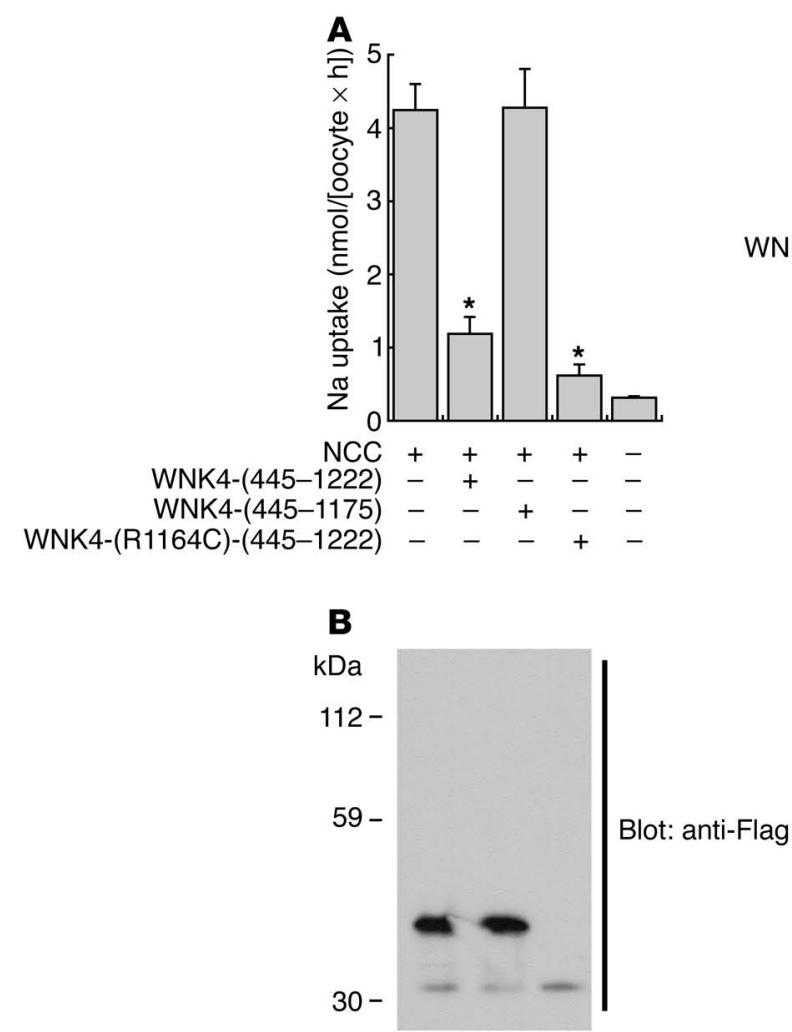
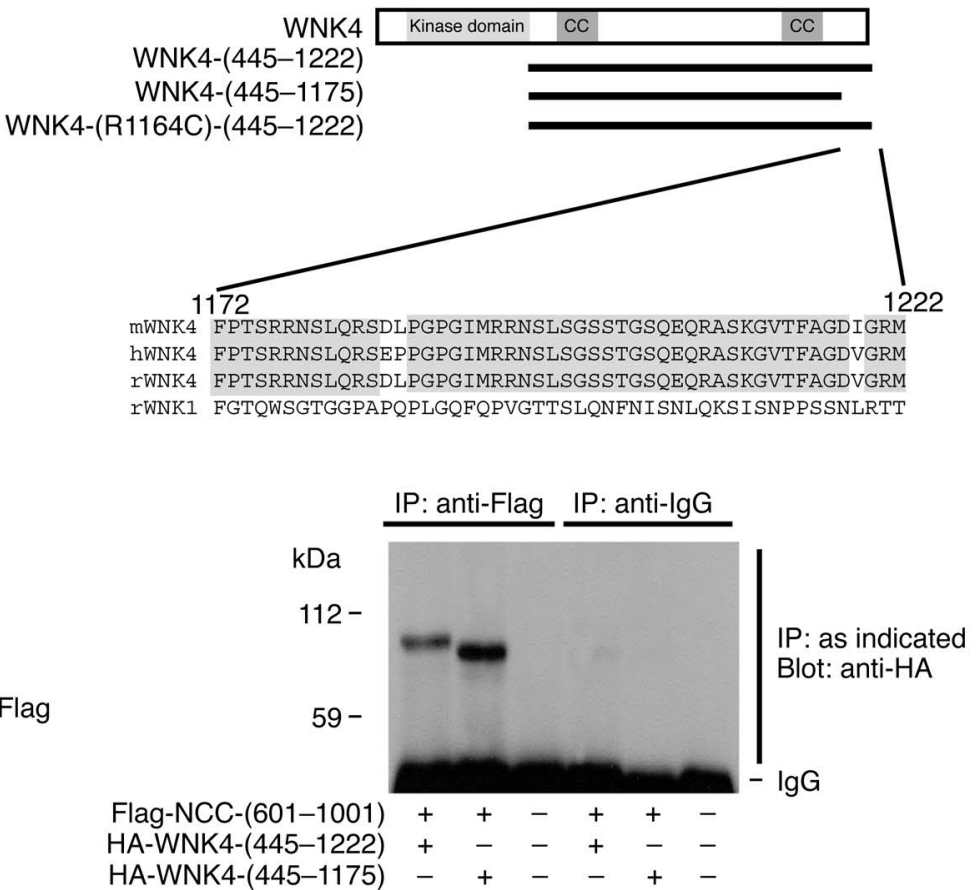

Figure 5

Localization of a negative regulatory signal region of WNK4. (A) Oocytes were injected with RNA encoding WNK4 constructs and NCC. The WNK4 constructs are diagrammed below. WNK4 missing the carboxyterminal 47 amino acids did not inhibit uptake, in contrast to constructs that contained these amino acid residues (the homologous regions of WNK4 from mouse [m], human [h], and rat [r] are shown below, with the terminal domain of rat WNK1 for comparison). A WNK4 fragment containing a human disease-causing mutation (WNK4-[R1164C]-[445-1222]) inhibited uptake as effectively as the wild type. ${ }^{*} P<0.05$ versus NCC alone. (B) Immunoprecipitation of carboxyterminal WNK4 fragments by NCC. Oocytes were injected with cRNA encoding a Flag-tagged carboxyterminal fragment of NCC and HA-tagged carboxyterminal WNK constructs. Both carboxyterminal WNK4 constructs were immunoprecipitated by anti-Flag (NCC) antibodies.

that WNK4 regulates ROMK through an effect on clathrin-coated vesicle-mediated endocytosis (16). The first goal of the present experiments, therefore, was to investigate mechanisms by which WNK4 regulates NCC activity. The current results show that the carboxyterminal 200 amino acids of NCC associate in a protein complex with WNK4; in contrast, the amino-terminal cytoplasmic domain of NCC does not interact with WNK4. These results extend those of Wilson and colleagues, who found that WNK4 associates with the carboxyterminal 416 amino acid residues of NCC (5).

Our results also indicate that the carboxyterminal 222 amino acids of WNK4 are sufficient to inhibit NCC activity. They further indicate that the carboxyterminal 47 amino acid residues of WNK4 are required to inhibit NCC activity, even though they are not essential for NCC binding. This suggests that the 47 carboxyterminal amino acid residues of WNK4 comprise an essential region for negative regulatory signaling. They also suggest that the association of WNK4 and NCC in a protein complex plays a role in regulating NCC membrane abundance; WNK4 constructs that inhibit NCC activity (such as WNK4-[808-1222]) (Figure 4B) bind to the transport protein avidly. These experiments emphasize that physical association may be necessary for functional effects but is not sufficient. Although Wilson and colleagues (5) reported that a putative kinasedead WNK4 (WNK4-[D318A]) does not inhibit NCC activity, the present results indicate that WNK4 kinase activity is not required to suppress NCC. To confirm this, a highly active WNK4 construct was mutated at amino acid 318. WNK4-(D318A)-(168-1222) inhibited NCC activity as efficiently as its wild-type homolog. Because a WNK4 fragment that does not include the kinase domain can fully inhibit NCC activity, it appears that WNK4 regulates NCC primarily via protein-protein interactions rather than by phosphorylation. Interestingly, introducing a mutation that causes FHHt into a truncated WNK4, WNK4-(R1164C)-(445-1222), did not affect its ability to inhibit NCC. This raises the possibility that interactions between WNK4, NCC, and another unidentified protein affect ion transport and emphasizes the potentially complicated WNK interactions that may underlie the human disease.

The carboxyl terminus of WNK4 was shown to associate in a protein complex with ROMK and be required to inhibit its membrane abundance (16). Because those experiments used a longer WNK4 construct that includes both coiled-coil domains, it is not clear whether the same WNK4 region interacts with both ROMK and NCC. Yet the observation that WNK4 mutations have disparate effects on NCC $(4,5)$ and ROMK (16), together with the evidence that clathrin-coated pit mechanisms regulate $\operatorname{ROMK}(16,22)$ but not NCC (5), suggest that the effects of WNK4 on transport proteins may involve distinct, yet physically contiguous, domains. 
A

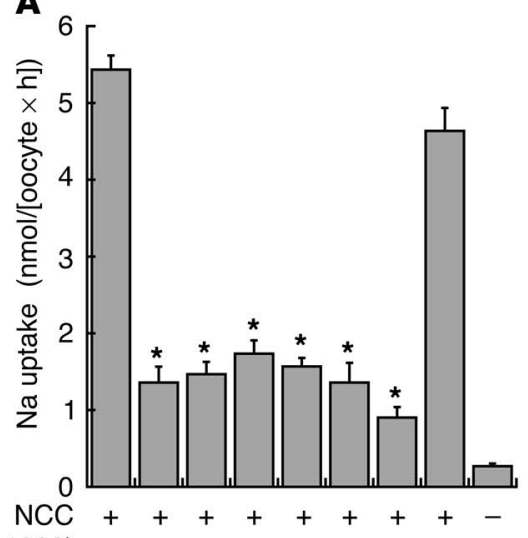

WNK4-(168-1222) -+-+++++-

WNK4-(D318A)-(168-1222) - - + - - - - -

WNK1-(1-491) - $-\quad+\quad-\quad-\quad-$

WNK1-(1-555) - ---+---

WNK1-(1-1036) - - - -+- -

WNK1-(640-2126) - $---_{-}-+-$

WNK1-(1-2126) - $----\quad-+-$

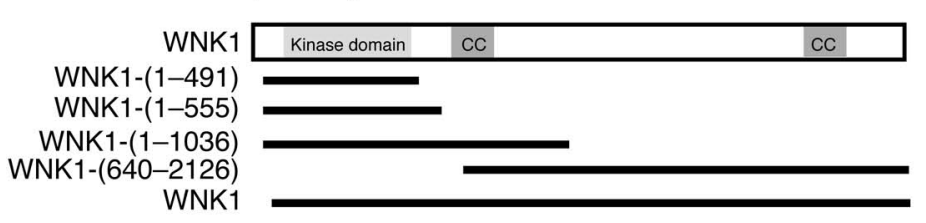

B

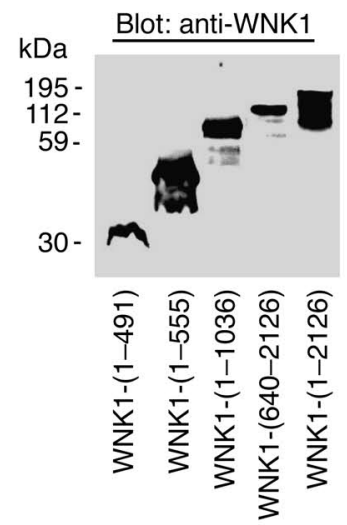

C

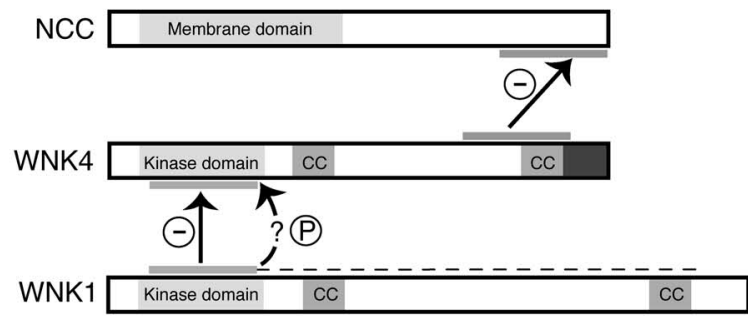

Figure 6

Only full-length WNK1 can inhibit WNK4 effects. (A) ${ }^{22}$ Na uptake by oocytes injected with cRNA encoding NCC, WNK4-(168-1222), WNK4(D318A)-(168-1222), and several WNK1 constructs, as indicated. The WNK1 constructs are diagrammed below. Note that a putative kinasedeficient WNK4 construct inhibited Na uptake as effectively as a wild-type product. Only the full-length WNK1 construct suppressed the effect of WNK4 on NCC. ${ }^{*} P<0.05$ versus NCC alone. (B) Blot showing that all WNK1 constructs were expressed by the oocytes at the protein level. (C) Schematic representation of major results. The carboxyl terminus of WNK4 associates with the carboxyl terminus of NCC. This interaction inhibits NCC activity (-). The 47 terminal WNK4 amino acids (shaded dark gray) function as a negative regulatory signal region but are not essential for binding. The amino-terminal domain of WNK1 associates with the amino-terminal domain of WNK4. This interaction inhibits WNK4 activity in a kinase-dependent manner. The functional effect requires full-length WNK1 (dotted line). Although catalytic activity is required, it is not clear whether WNK1 phosphorylates WNK4 directly.

The present results confirm our previous observation that WNK1 suppresses WNK4-mediated NCC inhibition and indicate that WNK1 and WNK4 can associate in a protein complex. They indicate that the interaction between WNK4 and WNK1 involves the amino-terminal (kinase) domains of each protein, regions that are highly homologous (1). The current results also indicate that this interaction between WNK1 and WNK4 is essential for WNK1 suppression of WNK4-mediated NCC inhibition. Interestingly, the amino terminus of WNK4 is required to interact with WNK1, even though the carboxyl terminus of WNK4 can inhibit NCC fully when expressed alone. These conclusions are illustrated schematically in Figure 6C. The current results also indicate that WNK1(D368A) is devoid of WNK4-inhibitory activity. This mutant was shown to lack kinase activity by $\mathrm{Xu}$ and colleagues (2), suggesting that both physical association and catalytic activity are required for WNK1 to inhibit WNK4 (as shown in Figure 6C). Partial-length WNK1 constructs, either amino-terminal or carboxyterminal, do not suppress WNK4 effects on NCC.

Our data reveal different patterns of regulation between WNK1 and WNK4. With regard to WNK4, deleting the kinase domain preserves the full inhibitory effect of the protein. Thus, WNK4mediated NCC inhibition appears to be strongly dependent on protein-protein interactions. In contrast, the WNK1 effect on WNK4 does not occur if the carboxyl terminus of WNK1 is expressed alone, or if a kinase-dead mutant is employed. Surprisingly, despite the requirement for kinase activity, WNK1 constructs that contain the kinase domain alone (WNK1-[1-491]) and WNK 1 constructs that contain the first coiled-coil domain (WNK1-[1-1036]) do not suppress WNK4-mediated NCC inhibition. This is despite the fact that this fragment has been demonstrated to possess full kinase activity in vitro $(7,8)$. One hypothesis to explain these results is that the WNK1 action on WNK4 may require tetramer formation (8). Deletion of the WNK1 carboxyl terminus would be expected to interfere with such multimer formation.

The observation that a kinase-dead WNK1 does not suppress WNK4-inhibiting activity may be relevant to the pathogenesis of FHHt. WNK1 is subject to complex regulatory alternative splicing, generating kinase-active and kinase-deficient WNK1 isoforms (9-11). In the kidney, the predominant isoform appears to contain exon $4 \mathrm{a}$ and exon 12 and to lack exons $1-4$ and exon 11; it is, therefore, kinase deficient $(9,10)$. This WNK1 isoform is generated by a separate promoter and by alternative splicing. There are also other renal WNK1 products. These include WNK1 proteins that 
lack exon 12, as well as kinase-active full-length WNK1 isoforms $(9,10)$. Although the effects of the kinase-deficient kidney-specific WNK1 isoform (KS-WNK1) have not been tested, it would be anticipated that this kinase-deficient kidney-specific WNK1 splice isoform would not inhibit WNK4, because WNK1 kinase activity is required (as shown in Figures 2 and 6). Under normal conditions, if the kinase-sufficient, full-length WNK1 is expressed at low levels along the distal nephron, then the ability of WNK4 to inhibit NCC activity will be manifest. If FHHt-causing WNK1 intronic mutations (1) increase expression of a kinase-sufficient WNK1 isoform along the distal nephron, this would suppress the constitutive effects of WNK4 on NCC, thereby increasing renal $\mathrm{Na}$ and $\mathrm{Cl}$ reabsorption and inhibiting $\mathrm{K}$ secretion. Although WNK4 has been shown to regulate ROMK (16), the effects of WNK1 isoforms on this transport protein have not been reported.

These data provide further support for the hypothesis that WNK1 and WNK4 converge, at least in part, on a common pathway in the distal nephron, where they regulate solute transport. As discussed above, WNK1 deficiency reduces blood pressure (3). The current results indicate that the ability to suppress WNK4 activity is generated by the catalytically active kinase domain of WNK1, but that the intact WNK1 protein is required for such an effect to occur. These observations raise the possibility that novel antihypertensive drugs may be developed that enhance WNK4-mediated effects on renal $\mathrm{NaCl}$ transport and on blood pressure.

\section{Methods}

DNA constructs. Mouse WNK4 and rat WNK1 were generated previously (4). Other constructs were generated by PCR or by site-directed mutagenesis using the QuickChange kit (Stratagene). Amino-terminal HA-tagged constructs were generated by PCR using an HA sequence (ATGTACCCATACGATGTTCCAGATTACGCT; amino acids MYPYDVPDYA). All sequences were confirmed by sequencing (Vollum Institute, Oregon Health \& Science University).

Antibodies. A mouse WNK4 antibody generated against amino acids 644-664 was described previously (4). An anti-WNK1 antibody was generously provided by Melanie Cobb (University of Texas Southwestern, Dallas, Texas, USA) (2). A second WNK1 antibody (Y-1606) was generated against a peptide corresponding to rat WNK1 amino acids 691-718 and an additional amino-terminal cysteine (CAPGHTVSSIQAQSQPHGV). The peptide was synthesized by the HHMI/Keck Biotechnology Resource Laboratory at Yale University, coupled to keyhole limpet hemocyanin with the Imject Maleimide Activated Immunogen Conjugation Kit (Pierce), and used to immunize guinea pigs (Pocono Rabbit Farm). The resulting serum was shown to recognize a protein of the expected size from oocytes injected with WNK1, but not WNK4 or NCC (see Figure 5B).

Na uptake. Na uptake experiments were performed as described previously (4) with some modifications (23). DNA template (NCC, WNK1, and WNK4) was linearized downstream from the $3^{\prime}$ untranslated region, using NotI or AclI and transcribed using T7 RNA polymerase (mMESSAGE mMACHINE; Ambion). Sorted Xenopus oocytes were injected with $50 \mathrm{nl}$ of water each and 5-10 ng each of NCC, WNK1, WNK4, or various truncated constructs. The same amount of RNA was always injected into all groups. After injection, oocytes were stored in Barth's storage solution for 3-5 days at $18^{\circ} \mathrm{C}$, with fresh solution changes daily.

Injected oocytes were transferred to $\mathrm{ND} 6 \mathrm{Cl}^{-}$-free solution $(96 \mathrm{mM}$ $\mathrm{Na}^{+}$isethionate, $2 \mathrm{mM} \mathrm{K}^{+}$gluconate, $1.8 \mathrm{mM} \mathrm{Ca}^{++}$gluconate, $1 \mathrm{mM} \mathrm{Mg}^{++}$ gluconate, $5 \mathrm{mM}$ HEPES, $2.5 \mathrm{mM} \mathrm{Na}^{+}$pyruvate, and $5 \mathrm{mg} / \mathrm{dl}$ gentamicin, $\mathrm{pH}$ 7.4) 24 hours prior to the uptake assay. Thirty minutes prior to the addition of uptake medium, the oocytes were added to $\mathrm{ND}^{-1} 6 \mathrm{Cl}^{-}$- and
K-free solution with inhibitors ( $1 \mathrm{mM}$ ouabain, $100 \mu \mathrm{M}$ amiloride, and $100 \mu \mathrm{M}$ bumetanide), according to the protocol of Gamba (24). The oocytes were then transferred to isotonic uptake medium $(58 \mathrm{mM} \mathrm{NaCl}$, $38 \mathrm{mM} \mathrm{N}$-methyl-D-glucamine, $2 \mathrm{mM} \mathrm{KCl}, 1.8 \mathrm{mM} \mathrm{CaCl}_{2}, 1 \mathrm{mM} \mathrm{MgCl}$, $5 \mathrm{mM}$ HEPES, with inhibitors, $\mathrm{pH} 7.4$ ) and $10 \mu \mathrm{Ci} / \mathrm{ml}$ final concentration of ${ }^{22} \mathrm{Na}$, and incubated at $30^{\circ} \mathrm{C}$ for 1 hour. The oocytes were washed 3-5 times with $1.5 \mathrm{ml}$ ice-cold isotonic uptake medium, lysed in $0.1 \% \mathrm{SDS}$, added to 5 $\mathrm{ml}$ of liquid scintillant (Hionic-Fluor; Packard), and counted for 2 minutes in a liquid scintillation counter. Fifteen to 30 oocytes were analyzed for each uptake condition during each experiment. Mean values reflect 3-6 experiments (each utilizing 15-30 oocytes per experimental condition). All animal experiments were approved by the Oregon Health \& Science University Institutional Animal Care and Use Committee.

Immunoblot. Groups of 20-30 oocytes injected 4 days prior with cRNA were transferred to Eppendorf tubes. Incubation buffer was then replaced with $20 \mu \mathrm{l}$ per oocyte of homogenization buffer $(20 \mathrm{mM}$ Tris-HCl, $\mathrm{pH}$ 7.6, $100 \mathrm{mM} \mathrm{NaCl}, 2 \% \mathrm{NP}-40$ with protease inhibitors) and the oocytes were lysed by repeated vortexing and pipeting. Cellular debris were pelleted at $3,600 \mathrm{~g}$ for 10 minutes, and the supernatant was centrifuged at $3,600 \mathrm{~g}$ 3 times to remove additional debris. Floating yolk was removed with a cotton-tipped swab. All centrifugation steps were performed at $4{ }^{\circ} \mathrm{C}$.

Immunoblotting was essentially as described previously (23). Oocyte homogenates were solubilized in sample buffer $(100 \mathrm{mM}$ Tris- $\mathrm{HCl}, \mathrm{pH} 7.6$, $3 \%$ glycerol, $2 \%$ SDS, and $0.01 \% \beta$-mercaptoethanol) and heated at $60^{\circ} \mathrm{C}$ for 10 minutes. The proteins were then separated on a 3-8\% NuPAGE Tris-Acetate Gel (Novex; Invitrogen Corp.) and transferred to PVDF paper. Blots were blocked with Blotto-T (5\% nonfat dried milk in PBS-T) and incubated with the primary antibody. The blot was then washed, incubated with secondary antibody (HRP-coupled, diluted 1:2000; Zymed), and detected using the ECL+ kit (Amersham LIFE SCIENCE) in accordance with the manufacturer's instructions.

Immunoprecipitation. Oocytes were prepared as described above for Western blot. Primary antibody $(2 \mu \mathrm{l})$ was added to oocyte homogenate $(10$ oocytes in $600 \mu \mathrm{l}$ ) and incubated at room temperature for 1 hour (or at $4{ }^{\circ} \mathrm{C}$ overnight). Thirty microliters of protein A sepharose (Amersham Biosciences) were added and incubated at $4^{\circ} \mathrm{C}$ overnight. The reaction mixture was then centrifuged and the beads were washed 5 times in homogenizing buffer. Immunoprecipitated protein was eluted from the protein A sepharose by heating the mixture to $100^{\circ} \mathrm{C}$ for 10 minutes with sample buffer containing $2.5 \%$ SDS and $2.5 \% \beta$-mercaptoethanol. Immunoprecipitates were analyzed by SDS-PAGE and blotted, as described above.

Transient transfections and immunoprecipitation. HEK293T cells were cultured at $37^{\circ} \mathrm{C}$ with $5 \% \mathrm{CO}_{2}$ in Vitacell MEM (ATCC) supplemented with $2 \mathrm{mM}$ streptomycin/penicillin and $10 \%$ fetal calf serum. Transient transfections were performed using SuperFect Transfection Reagent (Qiagen) in accordance with the manufacturer's instructions. After transfection, cells were incubated for 48-72 hours. Cells were treated with $100 \mathrm{mM} \mathrm{NaCl}$ for 10 minutes before harvest. Cells were then washed in cold PBS and lysed at $4^{\circ} \mathrm{C}$ in lysis buffer $\left(10 \mathrm{mM}\right.$ Tris $\mathrm{HCl}, \mathrm{pH} 8.0,2.5 \mathrm{mM} \mathrm{MgCl}_{2}, 5 \mathrm{mM}$ EGTA, pH 8.0, 0.5\% triton X-100, $1 \mathrm{mM} \mathrm{Na}_{3} \mathrm{VO}_{4}, 50 \mathrm{mM} \mathrm{NaF}$, and $100 \mu \mathrm{l}$ protease inhibitor cocktail set III [Calbiochem] per $10 \mathrm{ml}$ of buffer). Lysates were cleared by centrifugation, and the supernatant was used for immunoprecipitation. The protein concentrations were determined by the Bradford method (Bio-Rad Laboratories). For each immunoprecipitation, extracts were incubated with $1 \mu \mathrm{g}$ of mouse monoclonal anti-HA (12CA5; Roche Diagnostics Corp.) or $10 \mu \mathrm{l}$ of rabbit polyclonal anti-WNK1/WNK4 for 2 hours or overnight at $4{ }^{\circ} \mathrm{C}$. The immunocomplex was precipitated by incubating with $40 \mu \mathrm{l}$ of $50 \%$ Protein G-sepharose slurry (Amersham Biosciences) for 1 hour at $4{ }^{\circ} \mathrm{C}$. Immunoprecipitates were washed 3 times with PBS. Bound protein was eluted by boiling for 10 minutes in $2 \times$ SDS 
sample buffer. Immunoprecipitates were analyzed by SDS-PAGE and blotted as described above.

Statistics. Group comparisons were by unpaired Student's $t$ test. When multiple comparisons were made, the Bonferroni correction was employed. A $P$ value less than 0.05 was considered significant.

\section{Acknowledgments}

This work was supported by the National Institute of Diabetes and Digestive and Kidney Diseases (NIH grant RO1 DK51496), by a Department of Veterans Affairs Merit Review, and by internal funds provided by Oregon Health \& Science University. The authors thank David Rosansky for helpful discussions, Bing-e Xu and Melanie Cobb for the WNK1 antibody, and the reviewers for strengthening the manuscript.

1. Wilson, F.H., et al. 2001. Human hypertension caused by mutations in WNK kinases. Science. 293:1107-1112.

2. Xu, B., et al. 2000. WNK1, a novel mammalian serine/ threonine protein kinase lacking the catalytic lysine in subdomain II. J. Biol. Chem. 275:16795-16801.

3. Zambrowicz, B.P., et al. 2003. Wnk1 kinase deficiency lowers blood pressure in mice: a gene-trap screen to identify potential targets for therapeutic intervention. Proc. Natl. Acad. Sci. U. S. A. 100:14109-14114.

4. Yang, C.L., Angell, J., Mitchell, R., and Ellison, D.H. 2003. WNK kinases regulate thiazide-sensitive $\mathrm{Na}-\mathrm{Cl}$ cotransport. J. Clin. Invest. 111:1039-1045.

5. Wilson, F.H., et al. 2003. Molecular pathogenesis of inherited hypertension with hyperkalemia: the $\mathrm{Na}-\mathrm{Cl}$ cotransporter is inhibited by wild-type but not mutant WNK4. Proc. Natl. Acad. Sci. U. S. A. 100:680-684.

6. Xu, B.E., et al. 2004. WNK1 activates ERK5 by an MEKK2/3-dependent mechanism. J. Biol. Chem. 279:7826-7831.

7. Wang, Z., Yang, C.L., and Ellison, D.H. 2004. Comparison of WNK4 and WNK1 kinase and inhibiting activities. Biochem. Biophys. Res. Commun. 317:939-944.

8. Xu, B.E., et al. 2002. Regulation of WNK1 by an autoinhibitory domain and autophosphorylation. J. Biol. Chem. 277:48456-48462.

9. O'Reilly, M., Marshall, E., Speirs, H.J., and Brown, R.W. 2003. WNK1, a gene within a novel blood
Received for publication June 16, 2004, and accepted in revised form February 15, 2005.

Address correspondence to: David H. Ellison, Division of Nephrology and Hypertension, Oregon Health \& Science University PP262, 3314 SW US Veterans Hospital Road, Portland, Oregon 97239, USA. Phone: (503) 494-8490; Fax: (503) 494-5330; E-mail: ellisond@ohsu.edu.

Portions of this work were presented at the American Society of Nephrology Meeting in San Diego, California, USA, on November 14-17, 2003, and at the American Society of Nephrology Meeting in St. Louis, Missouri, USA, on October 30, 2004. Portions of this work have appeared in abstract form (2003. J. Am. Soc. Nephrol. 13:75A). pressure control pathway, tissue-specifically generates radically different isoforms with and without a kinase domain. J. Am. Soc. Nephrol. 14:2447-2456.

10. Delaloy, C., et al. 2003. Multiple promoters in the WNK1 gene: one controls expression of a kidneyspecific kinase-defective isoform. Mol. Cell. Biol. 23:9208-9221.

11. Xu, Q., Modrek, B., and Lee, C. 2002. Genomewide detection of tissue-specific alternative splicing in the human transcriptome. Nucleic Acids Res. 30:3754-3766.

12. Morrison, D.K., Murakami, M.S., and Cleghon, V. 2000. Protein kinases and phosphatases in the Drosophila genome. J. Cell Biol. 150:F57-F62.

13. Nakamichi, N., et al. 2002. Compilation and characterization of a novel WNK family of protein kinases in Arabiodpsis thaliana with reference to circadian rhythms. Biosci. Biotechnol. Biochem. 66:2429-2436.

14. Verissimo, F., and Jordan, P. 2001. WNK kinases, a novel protein kinase subfamily in multi-cellular organisms. Oncogene. 20:5562-5569.

15. Choate, K.A., Kahle, K.T., Wilson, F.H., Nelson-Williams, C., and Lifton, R.P. 2003. WNK1, a kinase mutated in inherited hypertension with hyperkalemia, localizes to diverse Cl- -transporting epithelia. Proc. Natl. Acad. Sci. U. S. A. 100:663-668.

16. Kahle, K.T., et al. 2003. WNK4 regulates the balance between renal $\mathrm{NaCl}$ reabsorption and $\mathrm{K}+$ secretion. Nat. Genet. 35:372-376.

17. Kahle, K.T., et al. 2004. WNK4 regulates apical and basolateral Cl- flux in extrarenal epithelia. Proc. Natl. Acad. Sci. U. S. A. 101:2064-2069.

18. Yamauchi, K., et al. 2004. Disease-causing mutant WNK4 increases paracellular chloride permeability and phosphorylates claudins. Proc. Natl. Acad. Sci. U. S. A. 101:4690-4694.

19. Kahle, K.T., et al. 2004. Paracellular Cl- permeability is regulated by WNK4 kinase: insight into normal physiology and hypertension. Proc. Natl. Acad. Sci. U. S. A. 101:14877-14882.

20. Achard, J.M., Disse-Nicodeme, S., Fiquet-Kempf, B., and Jeunemaitre, X. 2001. Phenotypic and genetic heterogeneity of familial hyperkalaemic hypertension (Gordon syndrome). Clin. Exp. Pharmacol. Physiol. 28:1048-1052.

21. Achard, J.M., et al. 2003. Familial hyperkalemic hypertension: phenotypic analysis in a large family with the WNK1 deletion mutation. Am. J. Med. 114:495-498.

22. Zeng, W.Z., et al. 2002. Evidence for endocytosis of ROMK potassium channel via clathrin-coated vesicles. Am. J. Physiol. Renal Physiol. 283:F630-F639.

23. Kunchaparty, S., et al. 1999. Defective processing and expression of the thiazide-sensitive $\mathrm{Na}-\mathrm{Cl}$ cotransporter as a cause Gitelman's Syndrome. Am. J. Physiol. 277:F643-F649.

24. Gamba, G., et al. 1994. Molecular cloning, primary structure, and characterization of two members of the mammalian electroneutral sodium-(potassium)-chloride cotransporter family expressed in kidney. J. Biol. Chem. 269:17713-17722. 